\title{
Optimising Therapeutic Options for Patients with Advanced Pancreatic Neuroendocrine Tumours
}

\author{
James $\mathrm{YaO}^{1}$ and Alexandria T Phan² \\ 1. Associate Professor and Deputy Chair; 2. Gastrointestinal Medical Oncology, Associate Professor, \\ The University of Texas, MD Anderson Cancer Center, Houston, Texas, US
}

\begin{abstract}
Pancreatic neuroendocrine tumours (DNETS) are a rare form of pancreatic cancer. Several therapeutic options exist for pNETS; however, there is no algorithm to determine the optimum sequence of therapies. Approved treatments for pNETs include somatostatin analogues (SSAs), streptozocin-based chemotherapy and targeted therapies such as everolimus and sunitinib. Unapproved therapies include systemic peptide receptor-targeted radiotherapy (PRRT), temozolomide-based chemotherapy, liver resection, liver transplantation, hepatic artery embolisation with or without chemotherapy and selective internal radiation therapy (SIRT). An individualised approach to the treatment of pNETS is described. Firstly, it is necessary to decide whether it is appropriate to treat at all. For those with symptoms, it is necessary to define the treatment goal: symptomatic or oncological control. Symptoms may direct treatment decisions; for example in patients with hypogycaemia, everolimus would be the most effective therapy. In high-volume disease where tumour reduction is the highest priority, streptozocin-based chemotherapy would be a more appropriate choice. For patients with disease progression and a moderate-to-high tumour volume, targeted therapy is the preferred choice. Following the failure of first-line therapies, second-line options include other targeted agents and cytotoxic chemotherapy. PRRT is recommended only after failure of prior therapy. Treatment decisions of pNETS should be made in a patient-oriented manner and on a case-by-case basis.
\end{abstract}

\section{Keywords}

Pancreatic neuroendocrine tumour, pNET, everolimus, sunitinib, RADIANT-1, RADIANT-3, somatostatin analogues, streptozocin-based chemotherapy

Disclosure: James C Yao has acted as Consultant for Ipsen, Novartis and Pfizer, and has received research funding from Novartis. Alexandria T Phan has received research funding from Ipsen, Novartis and Lexicon.

Received: 9 August 2012 Accepted: 1 October 2012 Citation: European Oncology and Haematology, 2012;8(4):217-23 DOl: 10.17925/EOH.2012.08.4.217

Correspondence: James Yao, The University of Texas, MD Anderson Cancer Center, Houston, Texas, US. E: jyao@mdanderson.org

Support: The publication of this article was funded by Novartis. The views and opinions expressed are those of the authors and not necessarily those of Novartis.

Pancreatic neuroendocrine tumours (pNETs), also known as islet cell tumours, arise from the pancreatic islet of Langerhans and can be divided into functioning and non-functioning tumours based on whether they are associated with hormonal syndromes caused by excess hormone or peptide secretion. Functioning PNETs can be responsible for a variety of clinical syndromes: Zollinger-Ellison syndrome is caused by gastrinomas (tumours that oversecrete gastrin), insulinomas are pNETs that overproduce insulin or proinsulin and glucagonomas overproduce glucagon and enteroglucagon. Other hormonal syndrome tumour types include: vasoactive intestinal peptide-producing tumours (VIPomas), pancreatic polypeptidomas (PPomas), and somatostatinomas.

PNETs account for only $1.3 \%$ of all pancreatic cancer. ${ }^{2}$ Estimates of incidence vary but the most recent published data suggest an annual incidence of 1-3 per million individuals, per year. ${ }^{3-6}$ Data from 1,185 cases of pNETs from the surveillance, epidemiology and end results (SEER) database was used to further examine the epidemiology of this tumour type. Distribution of cancer stage for pNETs at diagnosis included $14 \%$ localised, $23 \%$ regional and $54 \%$ distant or metastatic. ${ }^{2}$ The median survival rate for patients with localised pNETs was not reached, however, the 5 -year survival rate was $79 \%{ }^{6}$ in patients with regional stage disease, the median survival was 111 months and the five-year survival rate was $62 \%$. These numbers were further reduced in patients with distant metastatic disease, with a median survival rate of 27 months and a five-year survival rate of $27 \%$.

Although the majority of pNETs occur sporadically, pNETs can arise in association with several hereditary cancer syndromes. Approximately $10 \%$ may be connected with multiple endocrine neoplasia type 1 (MEN1), an autosomal dominant inherited disorder characterised by mutations in the menin tumour suppressor gene and development of tumours of the pancreas, parathyroid and pituitary. In addition to MEN1, other genetic cancer syndromes associated with pNETs include: von Hippel-Lindau $(\mathrm{VHL})$ disease, tuberous sclerosis and neurofibromatosis. A recent study determined the exomic sequences of ten non-familial pNETs to explore the genetic basis of sporadic disease. ${ }^{8}$ The commonly mutated genes were then screened against an additional 58 pNETs and it was determined that, within the 68 pNETs analysed, $43 \%$ had alterations in DAXX (death-domain associated protein) or ATRX (alpha thalassemia/mental retardation syndrome X-linked) which encode subunits of a transcription/chromatin remodelling complex. A total of $44 \%$ contained mutations in MEN1, the menin tumour suppressor gene and $14 \%$ had mutations affecting genes within the mammalian target of rapamycin (mTOR) pathway.

These molecular associations suggest three key pathways are involved in the development of pNETs. The first involves menin and its role in cell cycle 
regulation and tumourigenesis suppression. Menin potentially has pivotal roles in transcriptional regulation, DNA processing and repair and cytoskeletal integrity, however its involvement in the inhibition of cell cycle is one of the best-studied functions of this protein. ${ }^{9}$ The second pathway involves the ATRX and DAXX genes, which form subunits of a nuclear protein involved in chromatin remodelling at telomeric and percentromeric regions. Mutations in these genes have been shown to correlate with the alternative lengthening of telomeres (ALT) phenotype..$^{10}$ The third pathway is regulated by the mTOR serine/threonine kinase, a downstream effector of the PI3K/AKT pathway. ${ }^{11}$ mTOR is involved in the regulation of tumour cell growth, proliferation, angiogenesis and apoptosis. In addition, mTOR integrates multiple upstream signals from the vascular endothelial growth factor (VEGF) and insulin-like growth factor (IGF) signalling pathways.

In recent years, an increasing number of therapeutic options have become available for the treatment of PNETs, however there are limited amounts of data available to guide which treatment should be used to begin therapy and how to sequence the therapeutic options. The current options for the treatment of PNETs include: somatostatin analogues (SSAS), streptozocin-based chemotherapy, everolimus and sunitinib. Other treatment options still lacking support from pivotal randomised clinical trials include: systemic peptide receptor-targeted radiotherapy (PRRT), temozolomide-based chemotherapy, SSAs for tumour control, liver resection, liver transplantation, hepatic artery embolisation with or without chemotherapy, and selective internal radiation therapy (SIRT), also known as radioembolisation. The goal of this article is to review the strength and weakness of each option and provide guidance on how best to match treatment options with individual patients.

\section{Approved Treatment Options}

\section{Somatostatin Analogues}

Since the late 1980s, SSAs have been employed for the management of functional pNETs. ${ }^{12}$ Activation of somatostatin receptors by SSAs results in inhibition of hormone and peptide secretion and therefore SSAs are the first-line treatment for functioning PNETs. SSAs are generally effective in the treatment of VIPomas, ${ }^{13}$ gastrinomas ${ }^{14}$ and glucagonomas..$^{15}$ Care is needed, however, when using SSAs against insulinomas, as they have the potential to suppress glucagon secretion and worsen hypoglycaemia.? In addition to symptomatic control, SSAs have been demonstrated to have an antitumour effect when employed in the treatment of midgut NETs. ${ }^{16}$ However, the antiproliferative property of SSAs in terms of progression-free survival in PNETs remains unanswered.

\section{Streptozocin-based Chemotherapy}

Currently, streptozocin (usually in combination with doxorubicin and/or 5-fluorouracil) is the standard cytotoxic chemotherapy regime for non-functioning GEP-NETs of pancreatic origin;17 however, the meaningful clinical benefit of this chemotherapeutic treatment is debatable. Response rates ranging from 6 to $69 \%$ have been reported for streptozocin-based chemotherapy. ${ }^{18-20}$ A Phase III trial published in 1992 that investigated the combination of streptozocin and doxorubicin, when compared with streptozocin plus fluorouracil, or chlorozotocin, found that streptozocin plus doxorubicin had a significant advantage in terms of survival (median, 2.2 versus 1.4 years; $p=0.004$ ) (see Table 1). ${ }^{20}$ The reported tumour response rate for streptozocin and doxorubicin was $69 \%$, and was due in part to the historical use of non-standard response rates, which differ from the currently used WHO/RECIST criteria. This use of non-standard response rates also means that there are no reliable data outlining progression-free survival rates in patients following treatment with streptozocin, alone or in combination.
In addition to the paucity of data demonstrating efficacy of this treatment regime, there is a lack of robust safety data (using modern criteria). Toxic reactions reported in the study by Moertel et al. included nausea and vomiting (experienced by $80-81 \%$ of those receiving streptozocin), stomatitis, diarrhoea, leucopenia, thrombocytopaenia, nephrotoxicity (as measured by creatinine elevation) and renal insufficiency (experienced in all treatment groups by $4-7 \%$ of participants). ${ }^{20}$ Nine patients in the study developed severe chronic renal insufficiency. In addition, three patients developed heart failure, possibility as a consequence of doxorubicin therapy. As many of the patients in the study did not respond to treatment and thus had only short exposures to treatment, this suggests that the potential for nephrotoxicity and cardiomyopathy may be underestimated. Patient selection is fundamental when deciding the sequence of therapies for the treatment of advanced pNETs, as alkylating agents such as streptozocin may increase the risk of secondary haematological malignancies when PRRT is given in the same patient.

\section{Everolimus}

Everolimus is an oral inhibitor of mTOR, a regulator involved in the control of cell proliferation. Inhibition of mTOR prevents phosphorylation of key regulatory proteins, resulting in G1 growth arrest. In addition, mTOR is involved in the mediation of downstream signalling pathways such as for VEGF and IGF which have been previously implicated in neuroendocrine tumour growth.

The first clinical trial of everolimus involved 30 patients with carcinoid tumours and 30 patients with pNETs who were treated with everolimus plus depot octreotide. ${ }^{21}$ The overall tumour response rate was $27 \%$ in the patients with pNETs. A follow-up Phase II study (RADIANT-1) enrolled a total of 160 patients with advanced pNETs who have failed prior chemotherapy, divided into two groups. The first included 115 patients who were treated with everolimus (10 $\mathrm{mg}$ daily), while the second group consisted of 45 patients treated with everolimus (10 mg daily) and depot octreotide (30 mg intramuscularly every 28 days). ${ }^{22}$ In group 1 there were partial responses in $9.6 \%$ of the patients, $67.8 \%$ with stable disease and $13.9 \%$ with progressive disease; median PFS was 9.7 months. In stratum 2, there were partial responses in $4.4 \%$, $80 \%$ with SD, and no patients with progressive disease; median PFS was 16.7 months. These results formed the basis of a Phase III trial of everolimus in patients with advanced pNETs.

RADIANT-3 was an international, double-blind, placebo-controlled Phase III trial and was the largest randomised study to be conducted in pNETs. A total of 410 patients with advanced pNETs and with documented disease progression prior to enrolment, were randomly assigned to receive either daily everolimus (10 mg) or placebo. The median PFS was 11 months in the group treated with everolimus, versus 4.6 months in the placebo group, representing a $65 \%$ reduction in the estimated risk of progression (hazard ratio for disease progression or death with everolimus, $0.35 ; 95 \% \mathrm{Cl}, 0.27$ to $0.45 ; p<0.001)$. The overall response rate (ORR) was $5 \%$ in those treated with everolimus versus $2 \%$ in the placebo group and suggested that the benefit gained from everolimus treatment with respect to increased progression-free survival was primarily due to disease stabilisation, minor tumour shrinkage or a lower incidence of progressive disease. No differences in overall survival (OS) were observed due to the preplanned crossover to everolimus following disease progression of those in the placebo arm. Median overall survival was not reached, but was $>32$ months at the time of analysis..$^{30}$ 
Table 1: Trial Results of Different Therapies for the Treatment of Pancreatic Neuroendocrine Tumours

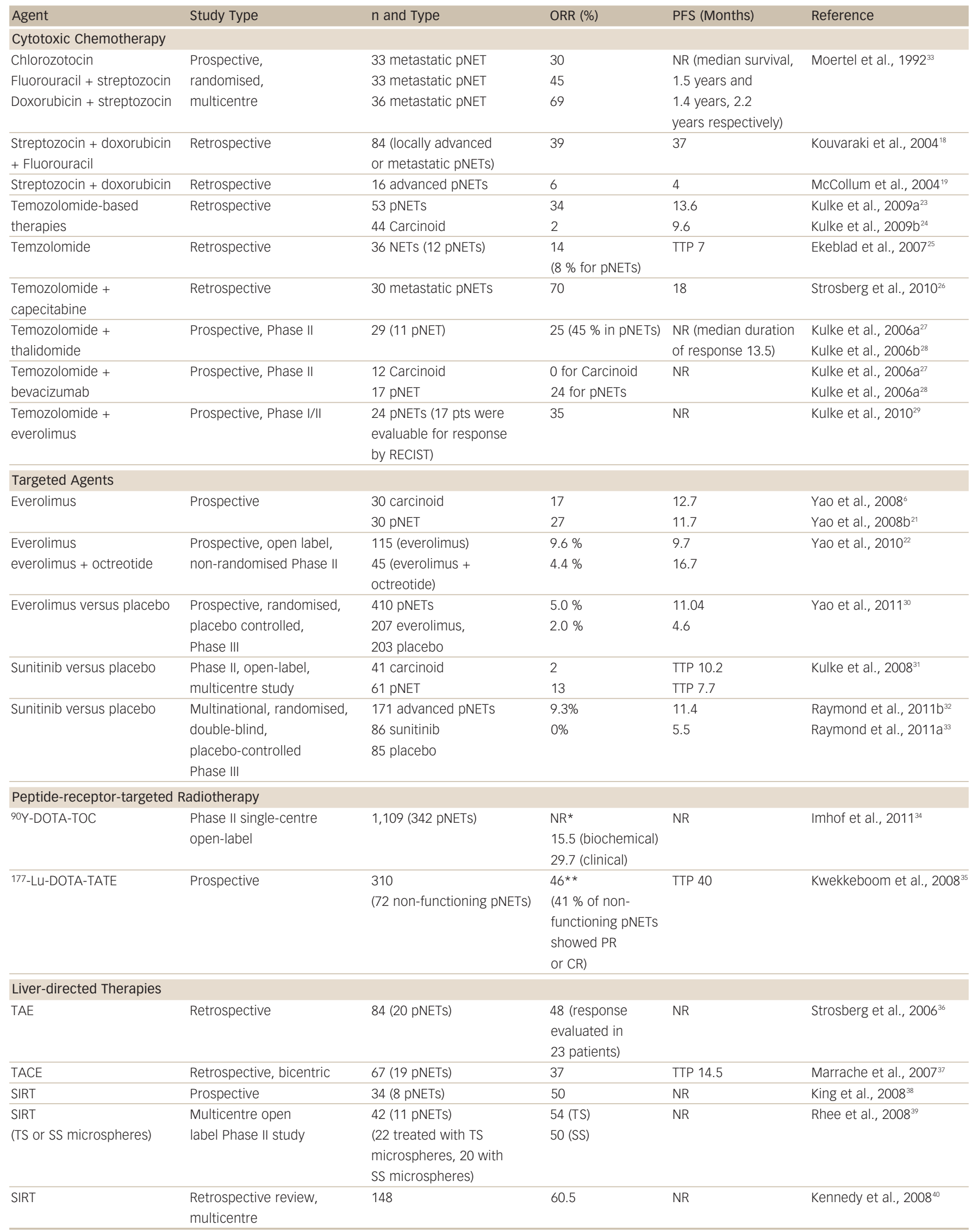

*Morphologic response rate as defined by any measurable decrease in the sum of the longest diameters was reported to be $34 \%$. **Comprising complete response, partial response and minor response. $N R=$ not reported; $O S=$ median overall survival; $P F S=$ progression-free survival; $P N E T S=$ pancreatic neuroendocrine tumours; $P t S=$ patients; $R R=$ response rate; $S D=$ stable disease; $S S=$ SIR-Spheres ${ }^{\circledR} ;$ TS $=$ TheraSphere ${ }^{\Theta} ;$ TTP $=$ time to progression; ${ }^{90} \mathrm{Y}$-DOTA-TOC $={ }^{177}$-Ytrium 1,4,7,10-tetraazacyclododecane-1,4,7,10-tetraacetic acid - tyrosine octreotide; LU-DOTA-TATE = Lutetium 1,4,7,10-tetraazacyclododecane-1,4,7,10-tetraacetic acid - tyrosine octreotate. 
Recent analysis of the OS of everolimus compared with placebo using a matching-adjusted indirect comparison of randomised trials found a significantly prolonged OS compared with placebo (hazard ratio [HR] for death $=0.61,95 \% \mathrm{Cl}=0.38-0.98, \mathrm{p}=0.04) .{ }^{41}$ Everolimus is now approved by the US Food and Drug Administration (FDA) and in the EU for the treatment of pNETS.

In addition to the demonstrated efficacy of everolimus, data suggest that everolimus treatment may reduce hormonal secretion from tumours. Furthermore, the use of everolimus in four patients with insulinoma and refractory hypoglycaemia was demonstrated to improve glycaemia control. ${ }^{23}$ Treatment with everolimus has also been noted to decrease serum levels of gastrin and glucagon in patients with pNET, ${ }^{42}$ as well as the important biomarkers chromogranin A (CgA) and neuron-specific enolase (NSE).

The safety data obtained in the RADIANT-3 trial are robust, based on mature follow-up and significant exposure duration. The most common side effects noted in the Phase III trial were stomatitis (64\%), rash (49\%), diarrhoea (34\%), fatigue (31\%), infections ( $23 \%$ ) and pneumonitis (12\%). The most frequent grade $3-4$ adverse events were stomatitis (seen in $7 \%$ of patients), anaemia (6\%), and hyperglycaemia (5\%), thrombocytopenia, diarrhoea, hypophosphatemia and neutropenia. Management of adverse events involved the use of antibiotics for patients with infections, while dose reduction, treatment-break along with glucocorticoids were administered in grade 3-4 non-infectious pneumonitis or interstitial lung disease.$^{30}$ Monitoring of renal function, blood glucose, lipids and haematological parameters is recommended prior to treatment and periodically thereafter.

\section{Sunitinib}

Angiogenesis is an important aspect of cancer progression, and proangiogenic factors such as VEGF and the VEGF receptor are highly expressed in pNETs. Sunitinib is an oral tyrosine kinase inhibitor of VEGF receptor, PDGR receptors and other tyrosine kinases including: c-kit, RET, CSF-1R, and FLT3, and has direct antitumour and antiangiogenic effects. In a Phase II clinical trial of 66 patients with advanced PNET $(n=66)$, the ORR was $16.7 \%$, $68 \%$ had stable disease and the median time to tumour progression was 7.7 months. ${ }^{31}$ These positive results were the basis of a Phase III, randomised, double-blind, trial of sunitinib versus placebo which was ended early, due to significant differences in disease progression and deaths in the placebo group..$^{33} \mathrm{~A}$ total of 171 patients were enrolled in the trial, 86 receiving sunitinib ( $37.5 \mathrm{mg}$ daily) and 85 receiving placebo. The median PFS in the sunitinib treatment was group was 11.4 months, compared with 5.5 months in the placebo group (HR 0.42; $95 \% \mathrm{Cl}$ $0.26-0.66 ; p<0.001)$. The ORR in the sunitinib treatment arm was $9 \%$, versus $0 \%$ in those treated with placebo. Based on these results, sunitinib was approved for treatment of unresectable or metastatic, well-differentiated pNETs by the FDA and European Commission.

Examination of the baseline patient characteristics from this Phase III trial shows some imbalance in prognostic factors, with differences in performance status and in the number of metastatic sites. ${ }^{33}$ In the sunitinib arm, $62 \%$ of patients had an ECOG performance status score of 0 and $38 \%$ had a score of 1 , while in the placebo arm $48 \%$ scored 0 and $51 \%$ scored 1 . Additionally, $28 \%$ of those in the sunitinib arm had more than three sites of disease, compared with $41 \%$ of those in the placebo arm. Early termination of the study may have led to an overestimation of efficacy (in comparison, the median time to progression [TTP] for sunitinib in a Phase II study was only 7.7 months). ${ }^{31,43}$ This also means that the false-positive rate was not controlled for and PFS did not cross the Lan-DeMets and O'Brien-Fleming efficacy boundary for statistical significance. In the initial report of the Phase III study, it was suggested that sunitinib provided a possible benefit in overall survival; ${ }^{33}$ however, analysis subsequent to a longer follow up period found no difference in overall survival between the sunitinib and placebo arms. ${ }^{32}$ The safety data obtained for sunitinib are not as robust as those obtained for everolimus due to the short exposure duration in the Phase III sunitinib trial. The most common side effects were diarrhoea (59\%), fatigue (32\%), nausea (45\%), asthenia (34 \%) and vomiting (34\%), while the most frequent grade 3-4 adverse events consisted of neutropenia (12\%), hypertension (10\%), hand-foot syndrome (6\%), and leukopenia (6\%). ${ }^{33}$ Sunitinib appears to be better tolerated when dosed at $37.5 \mathrm{mg} /$ day; at doses of $50 \mathrm{mg} /$ day, $89 \%$ of patients reported fatigue and $62.6 \%$ of patients had at least one dosing interruption. ${ }^{31}$ Although cardiotoxicity was not reported in either of these studies, it has been previously associated with sunitinib use ${ }^{44}$ and patients with cardiac risk factors should be closely monitored.

\section{Unapproved Options}

With a few exceptions, there is a paucity of large, randomised, prospective studies to evaluate the efficacy of these treatments and few approved treatments for pNETS. Although many of the following unapproved treatment options are used widely, there are scant data available to make evidence-based decisions on appropriate treatment choices.

\section{Peptide Receptor Radionuclide Therapy}

The majority of pNETs express somatostatin receptors and this allows their visualisation using radiolabelled SSAs such as OctreoScan ${ }^{\mathrm{TM}}$ (111/n-octreotide). PRRT utilises SSAS, which act as ligands for the radionuclides (indium-111 [111/n], yttrium-90 [90Y], or lutetium-177 [177Lu]) to actively target the somatostatin receptor expressing tumour. Studies have reported overall tumour response rates between 24 and $46 \%$ following PRRT ${ }^{35,45}$ and stable disease has been reported in up to $77 \%$ of patients. ${ }^{46}$ However, the different types of PRRTs have not been compared with each other or with other therapies, and randomised studies employing these therapies are lacking. In a study of 504 patients, 310 evaluable patients treated with 177-Lu-DOTA-TATE, the objective response rate of PNETs to PRRT treatment was $43 \%$ (CR $4.4 \%$ and PR $38.5 \%$ ) and was higher than NETs originating in the small intestine $22 \%$ (CR $1 \%$ and PR $22 \%$ ), however patients with gastrinoma, VIPoma, or insulinoma had significantly shorter response durations than other patients. ${ }^{35}$ However, the lack of intend-to-treat analysis and high rate of missing data (38\%) makes the results difficult to interpret. A recent Phase II trial in 1,109 patients with neuroendocrine tumours investigated the response to [90Y-DOTA]-TOC and of those enrolled $30.8 \%$ had pNETS. ${ }^{34}$ Unfortunately, the TTP and OS data from this report cannot be compared with other studies, as survival was calculated from diagnosis (which could have be many years earlier or when patient had early stage disease) and there was no comparator treatment or placebo group. Disease control was obtained in $39.3 \%$ of patients, the median TTP was 12.7 months. Treatment with [90Y-DOTA]-TOC, however, was associated with severe transient grade 3 to 4 haematological toxicities (affecting $12.8 \%$ of patients) and severe permanent renal toxicities (affecting $9.2 \%$ of patients). These safety issues may be partially related to the sites involved in uptake of the radiolabelled material and, as the distribution of material within the body can vary between patients, toxicity can be unpredictable.

\section{Temozolomide-based Chemotherapy}

Temozolomide is an oral cytotoxic alkylating agent, with a mechanism of action similar to that of streptozocin. Recent prospective and 
retrospective studies have suggested that temozolomide-based therapies may have similar efficacy to streptozocin-based treatments in patients with advanced PNETs. Results from retrospective studies have demonstrated tumour response rates to temozolomide chemotherapy ranging from 8 to $70 \%{ }^{25,26,28}$ Additionally, prospective studies employing temozolomide, in combination with thalidomide, bevacizumab, or everolimus, resulted in response rates of $24-45 \%{ }^{27-29}$ Currently there is limited available information on the optimal dosing regime and the relative efficacy of temozolomide used either in monotherapy or combination therapy has not been evaluated. Adverse events associated with the use of temozolomide include thrombocytopenia, lymphopenia, and opportunistic infections, and, similarly to streptozocin, temozolomide treatment may increase the risk of secondary haematological malignancies when PRRT is given in the same patient.

\section{Liver Resection}

Patients with pNETs frequently present with liver metastases, and surgical excision of such metastases should be considered where possible. Surgery may involve complete resection (resection of all visible hepatic tumours), or may simply involve palliative resection, which is especially beneficial in patients with functional hepatic metastases. If at least $90 \%$ of the visible tumour can be removed, the general practice is to employ surgical resection. Unfortunately the effect of hepatic resection on patient outcome has not been evaluated prospectively, however a retrospective study comprising 339 patients with NETs who underwent surgical management for liver metastases found that, although surgical resection was associated with increased survival (overall 5- and 10-year survival rates of 74 and $51 \%$, respectively), disease recurred in $94 \%$ of patients within five years. ${ }^{47}$ Reported survival rates for this surgical approach are $>60 \%$ at five years, which is double that of patients with untreated liver metastases. ${ }^{48,49}$

\section{Liver Transplantation}

Liver transplantation is considered an experimental treatment option with unproven benefit. ${ }^{50}$ In a retrospective study of 103 individuals who underwent orthotopic liver transplant (OLT) by Lehnert, the 2-year and 5-year survival was $60 \%$ and $47 \%$, respectively, however recurrence-free 5-year survival did not exceed $24 \% .^{51}$ It has been noted in several studies that PNETs have worse outcomes following OLT than more indolent NETS. ${ }^{51,52}$ As with other unapproved treatments for pNETs, further large prospective studies are warranted to demonstrate whether there is a substantial benefit to this treatment. OLT is not indicated for patients with pNETs based on the current level of evidence. ${ }^{53}$

\section{Hepatic Transarterial Embolisation and Chemoembolisation}

Hepatic transarterial embolisation (TAE) or transarterial chemoembolisation (TACE) are commonly used as a palliative treatment in patients who are not candidates for surgical resection and aim to induce tumour ischaemia based on the idea that hepatic tumours derive most of their blood supply from the hepatic artery, while healthy hepatocytes derive their blood supply from the portal vein. Using this technique, objective tumour responses have been noted in 33-67\% of patients. ${ }^{36,37,50,54}$ The wide variation of tumour response can be explained by the heterogeneous nature of the tumours, different patient exposures to cytotoxic agents and differences in hepatic burden. A disadvantage of this technique, is that patients require hospitalisation, with a recent study showing an average length of stay of four days following treatment..$^{55}$ specific complications following treatment include: post embolisation syndrome (vomiting, abdominal pain and fever), carcinoid crisis, hepatic abscess and acute liver failure. Contraindications to transarterial embolisation include: compromised portal vein, biliary enteric anastomosis and liver dysfunction.

\section{Selective Internal Radiation Therapy}

SIRT, also known as radioembolisation, consists of treatment with resin ${ }^{90} \mathrm{Y}$ microspheres in the hepatic artery, allowing delivery of high doses of radiation to the tumour, and is a preferred treatment choice over TAE or TACE for those with liver dysfunction or portal vein thrombosis. Studies have reported objective response rates of $50-63 \%{ }^{38-40}$ which are similar to those reported for other embolisation and chemoembolisation techniques. There appears to be less acute toxicity associated with SIRT as compared with embolisation or chemoembolisation, however the long-term safety is less well defined. Specific complications reported include: radiation-induced liver disease, hepatic abscess, acute pancreatitis and gastric ulceration.

\section{A Patient-oriented Treatment Strategy}

Treatment choices for patients with breast or colon cancer are straightforward due to the existence of extensive treatment algorithms, and therapeutic strategies for these cancers have been defined by multiple large randomised studies. This is not the case, however, in neuroendocrine cancers, where few large randomised studies have been completed and there is no algorithm to determine first-line treatment choice or the sequence of therapies thereafter. While some have made blanket recommendations about first-line therapy and others have simply stated that it is not possible to make an evidence-based recommendation for initial therapy, we advocate a patient-oriented approach.

\section{To Treat or Not to Treat?}

The first question to address when choosing a first-line treatment for patients with PNETs is: is treatment necessary? In patients who are fully asymptomatic, with low tumour burden, low proliferative rates and no observed progression, is there a need to treat? In a small proportion of patients with indolent pNETs disease is characterised by low tumour volume, low proliferative rates and low hormone and CgA marker levels. This type of patient may live for years without symptoms or measured cancer growth. In older patients, who have competing risk of death from other sources, active surveillance may be the best treatment option, as there is not the justification for exposure to the side effects and potential toxicity of long-term treatments.

\section{Treatment Goals}

A second question to address is: what is the treatment goal? There are two major determinants when choosing an appropriate first-line treatment: symptom control and oncological control.

If functional tumour symptoms are present, treatment must be chosen to best manage these symptoms. For example, if the patient is suffering from diarrhoea then SSAS should be employed, however if the patient is suffering from hypoglycaemia then everolimus would be a better therapeutic choice.

A third question is: what type of oncological control is needed for the patient (see Figure 2)? To answer this it is necessary to as further questions, for example, what is the tumour volume? How aggressive is the tumour? What is the proliferative (Ki 67/mitotic) rate? Is there radiological progression? It must also be remembered when choosing the initial therapy that there is a high likelihood that multiple treatments will be employed during the patient's illness. 
Figure 1: Schematic of Pathways Involved in Pancreatic Neuroendocrine Tumour Differentiation Key Pathways Identified during Analysis of pNET Mutations (A) and the Molecular Targets of Sunitinib and Everolimus (B)
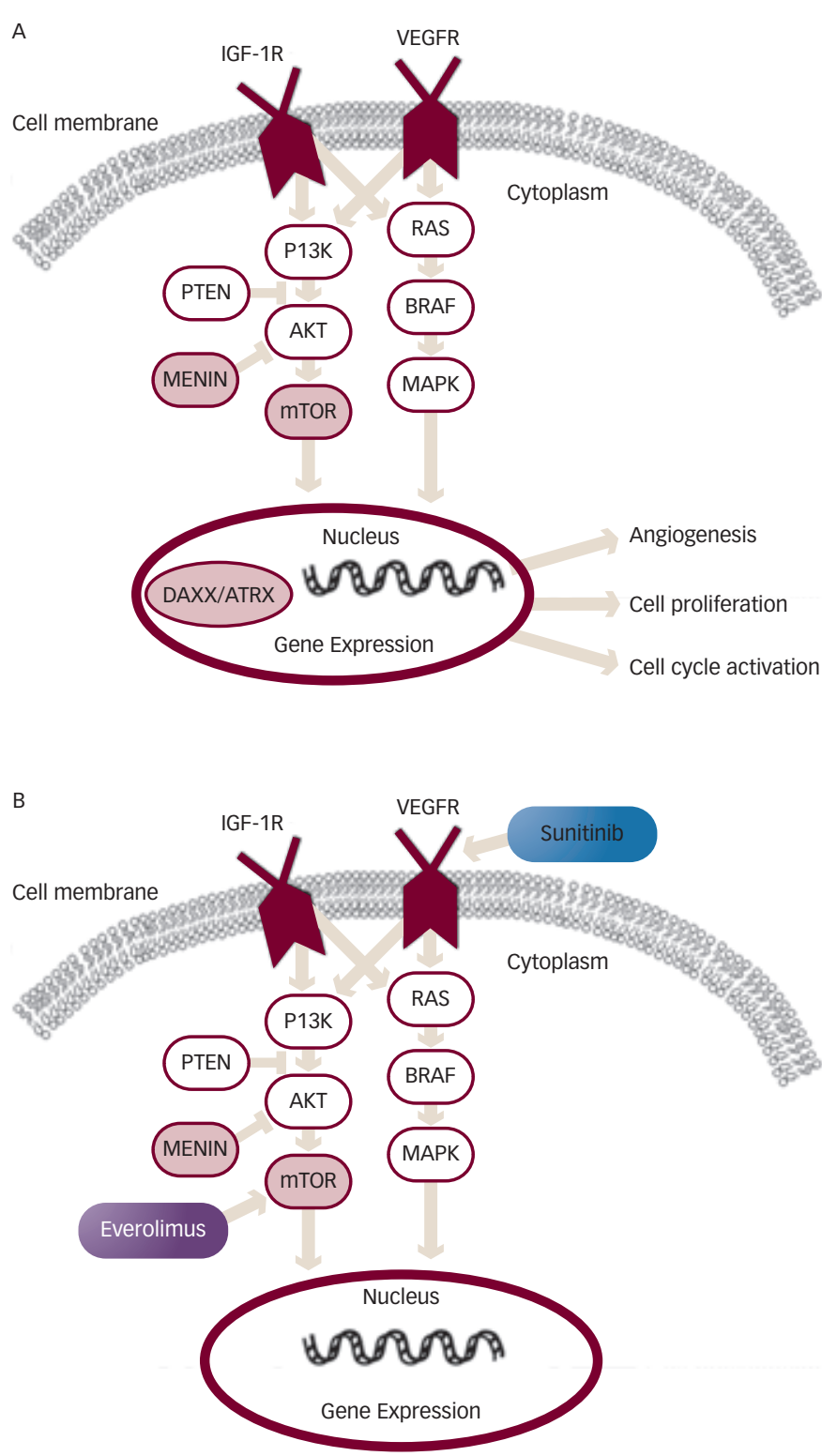

Three key pathways have been linked to the development of PNETs, involving MTOR, Menin or DAXX (death-domain associated protein) and ATRX (alpha thalassaemia/mental retardation syndrome $X$-linked) which encode subunits of a transcription/chromatin remodelling complex. Menin and mTOR both influence gene expression, and mutation in these pathways can result in an increase in angiogenesis, cell cycle activation, and cell proliferation. Mutations in the DAXX/ATRX genes have been shown to correlate with the alternative lengthening of telomeres (ALT) phenotype which is thought to increase cell proliferation. $A K T=$ also known as protein kinase $B ; \mid I G F-1 R=$ insulin-like growth factor 1; $M A P K=$ mitogen-activated protein kinase; $m T O R=$ mammalian target of rapamycin; PI3K = phosphatidylinositol 3-kinase; pNET; pancreatic neuroendocrine tumour; PTEN = phosphatase and tensin homologue; VEGFR = vascular endothelial growth factor receptor.

If tumour reduction is the top priority during treatment, streptozocinbased chemotherapy is favoured. This treatment is recommended for high volume disease where further progression would be life threatening.

For patients with disease progression, and tumours with an increased proliferative rate and moderate to high tumour volume, targeted therapy is the preferred option and the choice of everolimus or sunitinib should be based on the patient symptoms and co-morbidities. Everolimus is preferable if there are symptoms of hormonal secretion because this treatment has previously been demonstrated to reduce
Figure 2: Patient Characteristics Guide the Choice of First-line Therapy for Pancreatic Neuroendocrine Tumours

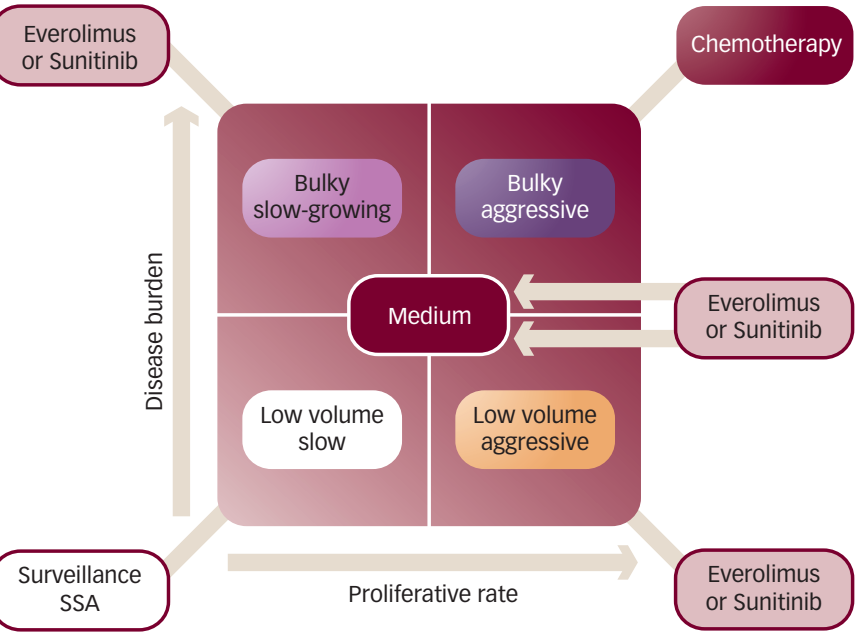

hormone and CgA levels ${ }^{42}$ and symptoms of hormonal secretion..$^{23}$ Use of everolimus is contraindicated if patients have poorly controlled diabetes or lung disease. Sunitinib should not be the first treatment choice if the patient has: poorly controlled hormonal symptoms, congestive heart failure, poorly controlled hypertension, high risk of a gastrointestinal bleed, or a history of myocardial infarction or stroke. In addition, an echocardiogram should be considered before use in patients who have been treated with prior cardiotoxic chemotherapy.

\section{Second-line Therapies}

Following failure of first-line treatment, the choices of second-line therapy include alternate targeted agents, and cytotoxic chemotherapy. PRRT is only recommended after failure of medical therapy by an ENETS expert consensus panel. ${ }^{56}$ Due to concern for long-term bone marrow and renal toxicities that may hinder therapeutic options, however, PRRT should be employed following failure of prior therapy. For patients with slowly progressive disease that reached high volume or with hormonal symptoms, hepatic artery embolisation, hepatic artery chemoembolisation, or SIRT is recommended.

\section{Conclusions}

There is currently an increasing number of therapeutic options available for the treatment of PNETs, however the data available to guide treatment choices are limited. The currently approved options for the treatment of pNETs include: SSAs, streptozocin-based chemotherapy, everolimus, and sunitinib, although SSAs are only used for the treatment of symptoms associated with functional tumours. The choice of first-line treatment for patients with well or moderately differentiated pNETS should be patient oriented, and factors such as tumour proliferative rate, and progression, age and the presence of co-morbidities, should guide treatment decisions. For some older patients with indolent tumours, active surveillance may prove the most beneficial treatment. Bulkier and more-aggressive tumours should be treated with streptozocin-based chemotherapy, while for other tumours where oncological control is needed everolimus or sunitinib should be employed. The choice between these targeted agents is dependent upon patient co-morbidities such as: symptoms of hormonal secretion, diabetes, or hypertension. Unlike other types of cancer, there are no treatment algorithms for selecting the appropriate therapeutic option for pNETS. Treatment management of these tumours must be decided in a patient-oriented manner and on a case-by-case basis. 
1. Oberg KE, The Management of Neuroendocrine Tumours: Current and Future Medical Therapy Options, Clin Oncol (R Coll Radiol), 2012;24:282-93.

2. Yao JC, Eisner MP, Leary C, et al., Population-based study of islet cell carcinoma, Ann Surg Oncol, 2007:14:3492-500.

3. Eriksson B, Oberg K, Neuroendocrine tumours of the pancreas, Br I Surg, 2000;87:129-31

4. Lam KY, Lo CY, Pancreatic endocrine tumour: a 22-year clinico-pathological experience with morphological, immunohistochemical observation and a review of the literature, Eur I Surg Oncol, 1997;23:36-42.

5. Oberg K, Castellano D, Current knowledge on diagnosis and staging of neuroendocrine tumors, Cancer Metastasis Rev, 2011;30(Suppl. 1):3-7.

6. Yao JC, Hassan M, Phan A, et al., One hundred years after "carcinoid": epidemiology of and prognostic factors for neuroendocrine tumors in 35,825 cases in the United States, $J$ Clin Oncol, 2008a;26:3063-72.

7. Kulke MH, Bendell J, Kvols L, et al., Evolving diagnostic and treatment strategies for pancreatic neuroendocrine tumors, J Hematol Oncol, 2011;4:29.

8. Jiao Y, Shi C, Edil BH, et al., DAXX/ATRX, MEN1, and mTOR pathway genes are frequently altered in pancreatic neuroendocrine tumors, Science, 2011;331:1199-203.

9. Wu T, Hua X, Menin represses tumorigenesis via repressing cell proliferation, Am I Cancer Res, 2011;1:726-39.

10. Heaphy $C M$, de Wilde RF, Jiao $Y$, et al., Altered telomeres in tumors with ATRX and DAXX mutations, science, 2011;333:425.

11. Populo $H$, Lopes $J M$, Soares $P$, The mTOR Signalling Pathway in Human Cancer, Int J Mol SCi, 2012;13:1886-918

12. Kvols LK, Buck M, Moertel CG, et al., Treatment of metastatic islet cell carcinoma with a somatostatin analogue (SMS 201-995), Ann Intern Med, 1987;107:162-8.

13. Kraenzlin ME, Ch'ng JL, Wood SM, et al., Long-term treatment of a VIPoma with somatostatin analogue resulting in remission of symptoms and possible shrinkage of metastases, Gastroenterology, 1985:88:185-7.

14. Shojamanesh H, Gibril F, Louie A, et al., Prospective study of the antitumor efficacy of long-term octreotide treatment in patients with progressive metastatic gastrinoma, Cancer, 2002; $94: 331-43$

15. Rosenbaum A, Flourie B, Chagnon S, et al., Octreotide (SMS 201-995) in the treatment of metastatic glucagonoma: report of one case and review of the literature, Digestion, 1989:42:116-20.

16. Rinke A, Muller $\mathrm{HH}$, Schade-Brittinger $\mathrm{C}$, et al. Placebo-controlled, double-blind, prospective, randomized study on the effect of octreotide LAR in the control of tumo growth in patients with metastatic neuroendocrine midgut tumors: a report from the PROMID Study Group, I Clin Oncol, 2009;27:4656-63

17. Falconi M, Bartsch DK, Eriksson B, et al., ENETS Consensus Guidelines for the management of patients with digestive neuroendocrine neoplasms of the digestive system: well-differentiated pancreatic non-functioning tumors, Neuroendocrinology, 2011:95:120-34

18. Kouvaraki MA, Ajani JA, Hoff P, et al., Fluorouracil, doxorubicin, and streptozocin in the treatment of patients with locally advanced and metastatic pancreatic endocrine carcinomas, J Clin Oncol, 2004;22:4762-71.

19. McCollum AD, Kulke MH, Ryan DP, et al., Lack of efficacy of streptozocin and doxorubicin in patients with advanced pancreatic endocrine tumors, Am I Clin Oncol, 2004;27:485-8.

20. Moertel CG, Lefkopoulo M, Lipsitz S, et al., Streptozocin-doxorubicin, streptozocin-fluorouracil or chlorozotocin in the treatment of advanced islet-cell carcinoma, N Engl J Med, 1992;326:519-23.
21. Yao JC, Lombard-Bohas C, Baudin E, et al., Daily oral everolimus activity in patients with metastatic pancreatic neuroendocrine tumors after failure of cytotoxic chemotherapy: a phase II trial, I Clin Oncol, 2010;28:69-76.

22. Yao JC, Phan AT, Chang DZ, et al. Efficacy of RAD001 (everolimus) and octreotide LAR in advanced low- to intermediate-grade neuroendocrine tumors: results of a phase II study, J Clin Oncol, 2008b;26:4311-8.

23. Kulke MH, Bergsland EK, Yao JC, Glycemic control in patients with insulinoma treated with everolimus, N Engl J Med, 2009a;360:195-7.

24. Kulke $\mathrm{MH}$, Hornick JL, Frauenhoffer $\mathrm{C}$, et al. 06-methylguanine DNA methyltransferase deficiency and response to temozolomide-based therapy in patients with neuroendocrine tumors, Clin Cancer Res, 2009b:15:338-45.

25. Ekeblad S, Sundin A, Janson ET, et al. Temozolomide as monotherapy is effective in treatment of advanced malignan neuroendocrine tumors, Clin Cancer Res, 2007;13:2986-91.

26. Strosberg JR, Fine RL, Choi J, et al., First-line chemotherapy with capecitabine and temozolomide in patients with metastatic pancreatic endocrine carcinomas, Cancer, 2010;117:268-75

27. Kulke $\mathrm{MH}$, Stuart $\mathrm{K}$, Earle $\mathrm{CC}$, et al., A phase II study of temozolomide and bevacizumab in patients with advanced neuroendocrine tumors, I Clin Oncol, 2006a;24:18S,4044.

28. Kulke MH, Stuart K, Enzinger PC, et al., Phase II study of temozolomide and thalidomide in patients with metastatic neuroendocrine tumors, J Clin Oncol, 2006b;24:401-6.

29. Kulke MH, Blaszkowsky LS, Zhu AX, et al., Phase I/II study of everolimus (RAD001) in combination with temozolomide (TMZ) in patients (pts) with advanced pancreatic neuroendocrine tumors (NET), Gastrointestinal Cancers Symposium, 2010;Abstract 223.

30. Yao JC, Shah MH, Ito T, et al., Everolimus for advanced pancreatic neuroendocrine tumors, N Engl I Med, 2011;364:514-23.

31. Kulke MH, Lenz HJ, Meropol NJ, et al., Activity of sunitinib in patients with advanced neuroendocrine tumors, $J$ Clin Oncol, 2008:26:3403-10

32. Raymond E, Niccoli P, Raoul J, et al., Updated overall survival and progression-free survival by blinded independent centra review of sunitinib versus placebo for patients with advanced unresectable pancreatic neuroendocrine tumours, I Clin Oncol, 2011b;29:Abstract 4008

33. Raymond E, Dahan L, Raoul JL, et al., Sunitinib malate for the reatment of pancreatic neuroendocrine tumors, N Eng/ / Med 2011a;364:501-13.

34. Imhof A, Brunner $\mathrm{P}$ Marincek $\mathrm{N}$, et al. Response, survival, and long-term toxicity after therapy with the radiolabeled somatostatin analogue [90Y-DOTA]-TOC in metastasized neuroendocrine cancers, J Clin Oncol, 2011;29:2416-23.

35. Kwekkeboom DJ, de Herder WW, Kam BL, et al., Treatment with the radiolabeled somatostatin analog [177 Lu-DOTA 0,Tyr3]octreotate: toxicity, efficacy, and survival, I Clin Oncol 2008;26:2124-30

36. Strosberg JR, Choi J, Cantor AB, et al., Selective hepatic artery embolization for treatment of patients with metastatic carcinoid and pancreatic endocrine tumors, Cancer Control, 2006:13:72-8.

37. Marrache F, Vullierme MP, Roy $C$, et al., Arterial phase enhancement and body mass index are predictors of response to chemoembolisation for liver metastases of endocrine tumours, Br J Cancer, 2007;96:49-55.

38. King J, Quinn R, Glenn DM, et al., Radioembolization with selective internal radiation microspheres for neuroendocrine liver metastases, Cancer, 2008;113:921-9.

39. Rhee TK, Lewandowski RJ, Liu DM, et al., 90Y Radioembolization for metastatic neuroendocrine liver tumors: preliminary results from a multi-institutional experience, Ann Surg, 2008;247:1029-35.

40. Kennedy AS, Dezarn WA, McNeillie P, et a ,

Radioembolization for unresectable neuroendocrine hepatic metastases using resin 90Y-microspheres: early results in 148 patients, Am I Clin Oncol, 2008:31:271-9.

41. Signorovitch JE, Swallow $E$, Kantor $E$, et al., Overall survival with everolimus, sunitinib, and placebo for advanced pancreatic neuroendocrine tumors: A matching-adjusted indirect comparison of clinical trials, I Clin Oncol, 2012;30(suppl.);abstract e14621.

42. De Vries EG, Anthony LB, Sideris L, et al., Effect of everolimus treatment on chromogranin A, neuron-specific enolase, gastrin, and glucagon levels in patients with advanced pancreatic neuroendocrine tumors (DNET): Phase III RADIANT-3 study results, I Clin Oncol, 2011;29(suppl.); ;abstract 10624.

43. Blumenthal GM, Cortazar P, Zhang JJ, et al., FDA approval summary: sunitinib for the treatment of progressive well-differentiated locally advanced or metastatic pancreatic neuroendocrine tumors, oncologist, 2012;17(8):1108-13. 44. Chu TF, Rupnick MA, Kerkela R, et al., Cardiotoxicity
associated with tyrosine kinase inhibitor sunitinib, Lancet, 2007;370:2011-9.

45. Waldherr C, Pless M, Maecke HR, et al., The clinical value of [90Y-DOTA]-D-Phe1-Tyr3-octreotide (90Y-DOTATOC) in the treatment of neuroendocrine tumours: a clinical phase study, Ann Oncol, 2001:12:941-5.

46. Cwikla JB, Sankowski A, Seklecka N, et al., Efficacy of radionuclide treatment DOTATATE Y-90 in patients with progressive metastatic gastroenteropancreatic neuroendocrine carcinomas (GEP-NETS): a phase II study, Ann Oncol, 2010;21:787-94.

47. Mayo SC, de Jong MC, Pulitano C, et al., Surgical management of hepatic neuroendocrine tumor metastasis: results from an international multi-institutional analysis, Ann Surg Oncol, 2010;17:3129-36.

48. Dousset B, Saint-Marc $\mathrm{O}$, Pitre J, Metastatic endocrine tumors: medical treatment, surgical resection, or liver transplantation, World I Surg, 1996;20:908-14.

49. Sarmiento JM, Heywood G, Rubin J, et al., Surgical treatment of neuroendocrine metastases to the liver: a plea for resection to increase survival, I Am Coll Surg, 2003;97:29-37.

50. Gulati AP, Saif MW, Is There a Role for Liver Transplantation in Metastatic Pancreatic Neuroendocrine Tumors (PNET)?, JOP, 2012;13:320-1

51. Lehnert $\mathrm{T}$, Liver transplantation for metastatic neuroendocrine carcinoma: an analysis of 103 patients, Transplantation, 1998:66:1307-12.

52. Le Treut YP, Delpero JR, Dousset B, et al., Results of liver transplantation in the treatment of metastatic neuroendocrine tumors. A 31-case French multicentric report, Ann Surg, 1997;225:355-64.

53. Alistar A, Sung M, Kim M, et al., Clinical Pathways for Pancreatic Neuroendocrine Tumors, I Gastrointest Cancer, 2012; in press.

54. Ruszniewski P, Rougier P, Roche A, et al., Hepatic arterial chemoembolization in patients with liver metastases of endocrine tumors. A prospective phase II study in 24 patients, Cancer, 1993:71:2624-30.

55. Lewis MA, Jaramillo S, Roberts L, et al., Hepatic artery embolization for neuroendocrine tumors: postprocedural management and complications, Oncologist, 2012;17:725-31.

56. Falconi M, Bartsch DK, Eriksson B, et al., ENETS Consensus Guidelines for the management of patients with digestive neuroendocrine neoplasms of the digestive system: well-differentiated pancreatic non-functioning tumors, Neuroendocrinology, 2012;95:120-34. 